\title{
Synchronized DSM Models ${ }^{\dagger}$
}

\author{
Jordi Bataller and Josep Bernabeu \\ Departamento de Sistemas Informaticos y Computacion \\ Universidad Politecnica de Valencia, Apdo. 22012, 46071 Valencia (Spain). \\ E-mail: \{bataller,josep\}@iti.upv.es, Tel. 346 3877069, Fax. 3463877359
}

\begin{abstract}
Memory accesses form a well understood paradigm for developing concurrent applications. Distributed Shared Memory systems, enable the creation of distributed applications based on shared memory accesses. A DSM system is characterized by the memory model it uses to perform memory accesses. There have been numerous models proposed over the years, and until recently there has been little attempt to provide a common formal framework to study their properties. DSM models can be roughly classified into synchronized (those which, in addition to usual read-write accesses, use special synchronization operations) and non-synchronized. In this paper we focus on the formalization of synchronized DSM models, extending a previous work on non-synchronized models.
\end{abstract}

\section{Introduction}

Shared memory access is a widely accepted paradigm to build concurrent applications. His main advantage is that it provides the programmer with a well-know communication paradigm. When implemented in a distributed system, programs do not have to take care of the details of the underlying communication system, potentially simplifying inter process communication. It would seem that using DSM would always offer worse performance than using the underlying message passing system. However, it has also been suggested the overheads incurred in implementing a given DSM model can be partially offset by appropriate compiler techniques [8] taking advantage of the locality of references. In any case, the worst performance shown by a given DSM system will depend on how strong is the memory model it implements. Strong memory models are easy to understand and use, incurring the highest cost. Weak models offer the best performance, at the price of a more obscure semantics. This has lead to the proposal of a large number of DSM models offering different tradeoffs between understandability and performance.

Unfortunately, memory models are frequently presented in operational terms, using intuitive concepts. In some cases special purpose formalisms are developed to present a particular model. Still, in other cases, the same authors use different

$\dagger$ This work has been supported in part by research grants TIC93-0304 and TIC960729 


\begin{tabular}{|l||l|}
\hline Symbol & Definition \\
\hline$\alpha \mid i$ & $\alpha$ subsequence containing operations matching $i:$ \\
\hline$\alpha \mid\left(t_{1}, t_{2}\right)$ & $\alpha$ subsequence containing operations of matching $t_{1}$ or matching $t_{2}$. \\
\hline$<_{t}^{\alpha}$ & ordering relation of type $t$ based on $\alpha$ sequence. \\
\hline$\left(<_{t}^{\alpha}\right) \mid i$ & subrelation of $<_{t}^{\alpha}$ formed by pairs of $<_{t}^{\alpha}$ whose operations match $i$. \\
\hline
\end{tabular}

Table 1. Naming conventions

formalisms in different works [14], [1] and [2]. This situation clearly leads to confusion, making it difficult to compare different proposals of memory models.

A formal framework to define memory models helps to study their properties and compare them to each other. In $[9,6]$ the authors show a formal framework, showing how to define most non-synchronized models with it. In this paper we extend that work to formalize synchronized memory models. As we will see, synchronized models extend a basic memory model (Slow consistency) by using special synchronization operations.

The rest of the paper is organized as follows. Section 2 summarizes the formal framework used in the paper. In section 3 we present our characterization of the synchronized models. Finally, section 4 we present some concluding remarks.

\section{Basic Concepts}

The formalism we use in this paper is a simple extension of the $\mathrm{I} / \mathrm{O}$ automata model introduced by Lynch [15]. Table 1 shows the naming conventions we follow in the paper. A memory model is characterized by the set of executions that a concrete implementation of the model is allowed to generate. An execution ${ }^{1}$ is an ordered sequence of single actions. The type of actions that can be issued are defined by the signature of the model. The signature of a memory model, $M(\mathcal{P}, \mathcal{V}, \mathcal{S})$, is the set $\{\operatorname{read}(i, x, v)$, write $(i, x, v)$, sync $(i, s, t)\}$ where $i \in \mathcal{P}$, $x \in \mathcal{V}_{D}, s \in \mathcal{V}_{S}, t \in S$ and $v^{2}$ is a valid value in the range of each variable; being $\mathcal{P}$ : set of processes, $\mathcal{V}$ : set of (shared) variables $\left(\mathcal{V}_{D}\right.$ : data variables, $\mathcal{V}_{S}$ : synchronization variables) and $\mathcal{S}=\{$ acquire, release $\}$ : set of synchronization types.

A model is defined by giving the property that an execution has to satisfy to be admissible by the model. That property has the form: "the execution $\alpha$ must admit a permutation of its actions respecting a given ordering $<_{?}^{\alpha}$ and being serial".

The orders we consider are summarized in table 2. All those orders are directly derived from two basic relations imposed by the actions of an execution: (1) Process Order (PO) is the order in which each process issues its actions. (2) writes-to is defined between a write action and the read action that gets the value written by the write action. Causal order is defined as the transitive closure of

\footnotetext{
${ }^{1}$ We use the term executions to refer to both executions and history sequences

${ }^{2}$ Without loss of generality, we use univalued writes (different writes write different values) to simplify the definition of some order relations.
} 


\begin{tabular}{|l||l|}
\hline Concept & Definition \\
\hline \hline$e_{1}<^{\alpha} e_{2}$ & holds if $e_{1}$ appears first than $e_{2}$ in $\alpha$ \\
\hline$e_{1} \mapsto e_{2}$ & $e_{1}$ writes to $e_{2}: e_{1}=$ write $(\cdot, x, a)$ and $e_{2}=\operatorname{read}(\cdot, x, a)$ \\
\hline$e_{1}<_{P O}^{\alpha} e_{2}$ & process order: $e_{1}<^{\alpha} e_{2}$ for some $i \in \mathcal{P}$. \\
\hline$e_{1}<_{C R}^{\alpha} e_{2}$ & causal relation: $e_{1}<_{P O}^{\alpha} e_{2}$ or $e_{1} \mapsto e_{2}$ or $\exists a: e_{1}<_{C R}^{\alpha} a<_{C R}^{\alpha} e_{2}$ \\
\hline $\begin{array}{l}\text { Serial } \\
\text { execution }\end{array}$ & $\alpha$ is a serial execution iff $\forall b=\operatorname{read}(\cdot, x, v)$ then $\exists a: a \mapsto b$ and $a<^{\alpha} b$ \\
\hline view $\left(\alpha,<_{?}^{\alpha}\right)$ & and $\nexists$ d $b=$ write $(\cdot, x, \cdot): a<^{\alpha} c<^{\alpha} b$ \\
\hline
\end{tabular}

Table 2. Ordering and concept definitions.

\begin{tabular}{|l||l|}
\hline Model & Definition \\
\hline \hline Sequential & $\exists \alpha^{\prime} \in$ view $\left(\alpha,<_{P O}^{\alpha}\right): \alpha^{\prime}$ is serial. \\
\hline PRAM & $\forall i \in \mathcal{P} \Rightarrow \exists \alpha^{\prime} \in$ view $\left(\alpha,<_{P O}^{\alpha} \mid(i\right.$, writes $\left.)\right): \alpha^{\prime}$ is serial. \\
\hline Cache & $\forall x \in \mathcal{V} \Rightarrow \exists \alpha^{\prime} \in$ view $\left(\alpha,<_{P O}^{\alpha} \mid x\right): \alpha^{\prime}$ is serial. \\
\hline Causal & $\forall i \in \mathcal{P} \Rightarrow \exists \alpha^{\prime} \in$ view $\left(\alpha,<_{C R}^{\alpha} \mid(i\right.$, writes $\left.)\right): \alpha^{\prime}$ is serial. \\
\hline Processor & $\forall i, j \in \mathcal{P} \Rightarrow \exists \alpha^{\prime} \in$ view $\left(\alpha,<_{P O}^{\alpha} \mid(i\right.$, writes $\left.)\right) \wedge \exists \alpha^{\prime \prime} \in$ view $\left(\alpha,<_{P O}^{\alpha}\right.$ \\
& $\mid \begin{array}{l}(j, \text { writes })): \alpha^{\prime} \text { and } \alpha^{\prime \prime} \text { are serial and } \forall x \in \mathcal{V} \text { then } \alpha^{\prime} \mid \text { write }(\cdot, x, \cdot)= \\
\\
\alpha^{\prime \prime} \mid \text { write }(\cdot, x, \cdot)\end{array}$ \\
\hline Slow & $\forall i \in \mathcal{P}, \forall x \in \mathcal{V} \Rightarrow \exists \alpha^{\prime} \in$ view $\left(\alpha,<_{P O}^{\alpha} \mid(i\right.$, write $(\cdot, x, \cdot)): \alpha^{\prime}$ is serial \\
\hline Local & $\forall i \in \mathcal{P} \Rightarrow \exists \alpha^{\prime} \in$ view $\left(\alpha,<_{P O}^{\alpha} \mid i\right) \alpha^{\prime}$ is serial \\
\hline
\end{tabular}

Table 3. Characterization of non-synchronized memory models.

the union of process order and writes-to relations. We use the notion of "serial execution" as the correctness criterion: an execution is said to be serial if every read returns the value stored by the immediately preceding write acting on the same variable. The notion of a permutation respecting a given ordering relation, is formalized as the "view" of a sequence. See these definitions in table 2.

\section{Synchronized Models Characterization}

Next, we give the formal definitions of the main memory models presented in the literature. Firstly, we group the definitions corresponding to non-synchronized models in table 3. See [9] for a more complete description.

The key feature of synchronized models is that they offer a basic coherence model (Slow) that can be strengthened by using synchronization actions. Synchronizations are also used to synchronize parallel processes.

Synchronizations act on so-called synchronizing variables that are guaranteed to be sequentially consistent. We distinguish special accesses (synchronizations), from ordinary accesses (or data) because many models are defined having synchronizations as a primitive operation. Synchronizations are further divided in acquires and releases. An acquire can be regarded as the request of a lock and a 


\begin{tabular}{|c|c|}
\hline Concept & Definition \\
\hline $\begin{array}{l}\text { Sync-well- } \\
\text { formedness }\end{array}$ & $\begin{array}{l}\alpha \text { is sync-well-formed iff } \\
(1) \forall i \in \mathcal{P}, \forall s \in \mathcal{V}_{S}:(\alpha \mid(i, s)) \mid(\text { acquire, release }) \text { is an empty sequence, or } \\
(2)\left(\alpha|(i, s)|(\text { acquire, release })=\text { sync }(i, s, \text { acquire }) \text { sync }(i, s, \text { release }) \alpha_{2}\right. \\
\text { and } \alpha_{2} \text { is sync-well-formed. } \\
\text { In this sequence, sync }(i, s, \text { acquire }) \text { corresponds to sync }(i, s, \text { release })\end{array}$ \\
\hline$\overline{D_{-}(s), D_{+}(s)}$ & $\begin{array}{l}\text { ordinary accesses that have to precede }(-) \text { or to follow }(+) \text { synchroniza- } \\
\text { tion } s \text {. }\end{array}$ \\
\hline$s_{1} \mapsto s_{2}$ & $\begin{array}{l}s_{1} \text { and } s_{2} \text { are synchronization operations of different processes such that } \\
\text { process issuing } s_{2} \text { obtains (reads) the access to the critical section granted } \\
\text { (written) by the process issuing } s_{1} \text {. }\end{array}$ \\
\hline$a<{ }_{D}^{\alpha} b$ & $\begin{array}{l}\text { iff (1) } a \text { is an ordinary access and } b \text { is a synchronization and } a \in D_{-}(b) \\
\text { or (2) } b \text { is an ordinary access and } a \text { is a synchronization and } b \in D_{+}(a)\end{array}$ \\
\hline$a<_{M B}^{\alpha}$ & iff $(1) a\left(<_{P O}^{\alpha} \mid \mathcal{V}_{\mathcal{S}}\right) b(a$ and $b$ are synchronizations $)$ or $(2) a<_{D}^{\alpha} b$ \\
\hline
\end{tabular}

Table 4. Concepts and orders related to synchronized models.

release as the its corresponding liberation. This definition imposes a well formed behaviour to be respected by each process: to issue acquire and release actions in an alternating way starting by an acquire, see table 4 .

The use of synchronizations introduce new relations among the actions of a sequence. They derive from the new basic relation sync-with. It is very similar to writes-to (in fact we use the same symbol). The synchronization, $s_{1}$, issued by a process granting access to the exclusion zone (a release synchronization) can be seen as writing a value read by the process receiving the grant by means of an acquire synchronization, $s_{2}$. Then, we say that $s_{1} \mapsto s_{2} . s_{1}$ is a release and $s_{2}$ is an acquire. The synch-with relation orders ordinary accesses from different processes, making it necessary to define which actions are ordered by any given synchronization, $s$. We represent by $D_{-}(s)$ and $D_{+}(s)$, the set of ordinary actions ordered preceding and following $s$ respectively, thus defining $<_{D}^{\alpha}$.

Using the above concepts and orderings (collected in table 4), we now describe the main synchronized DSM models. To that end, we partially cite each original definition and then argue our formal characterization. Table 5 groups our formal definitions of the synchronized models we are about to introduce.

Weak This model was introduced by Dubois et al. in [11]:

"In a multiprocessor system, storage accesses are weakly ordered if (1) accesses to global synchronizing variables are strongly ordered, (2) no access to a synchronizing variable is issued by a processor before all its preceding global data accesses have been globally performed, and (3) no access to global data is issued by a processor before its preceding accesses to a synchronizing variable has been globally performed". [...] (4) Between two consecutive operations on hardware recognized synchronization variables, no assumption can be made by the programmer of a process about the order in which stores are observed by other processes. 


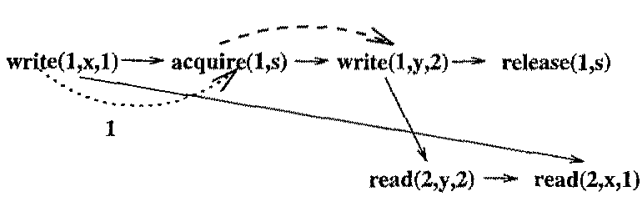

Process Order and Writes-to

Induced only by Weak model

$--->$ Induced both by Weak and Release models

Fig. 1. Release Execution. (view of $p_{1} ;$ write $(1, x, 1) \operatorname{read}(2, x, 1)$ acquire $(1, s)$ write $(1, y, 2)$ read $(2, y, 2)$ release $(1, s)$, view of $p_{2}:$ acquire $(1, s)$ write $(1, y, 2)$ read $(2, y, 2)$ write $(1, x, 1)$ read $(2, x, 1)$ release $(1, s))$. Dependence 1 disallows to be a Weak execution (it globally orders the writes of $p_{1}$ ).

\begin{tabular}{|c|c|}
\hline Model & Definition \\
\hline Weak & $\begin{array}{l}D_{-}(\operatorname{sync}(i, s, \cdot))=\left\{e: e<_{P O}^{\alpha} \operatorname{sync}(i, s, \cdot)\right\} \\
D_{+}(\operatorname{sync}(i, s, \cdot))=\left\{e: \operatorname{sync}(i, s, \cdot)<_{P O}^{\alpha} e\right\}\end{array}$ \\
\hline Release & $\begin{array}{l}D_{-}(\operatorname{sync}(i, s, \text { release }))=\left\{e: e<_{p O}^{\alpha} \text { sync }(i, s, \text { release })\right\} \\
D_{+}(\text {sync }(i, s, \text { acquire }))=\left\{e: \operatorname{sync}(i, s, \text { acquire })<_{P O}^{\alpha} e\right\}\end{array}$ \\
\hline $\begin{array}{l}\text { Lazy } \\
\text { Release }\end{array}$ & $\begin{array}{l}D_{-}(\operatorname{sync}(i, s, \text { acquire }))=\left\{e: \exists r e l \mapsto \operatorname{sync}(i, s, \text { acquire }) \text { and } e<_{P O}^{\alpha} \text { rel }\right\} \\
D_{+}(\operatorname{sync}(i, s, \text { acquire }))=\left\{e: \operatorname{sync}(i, s, \text { acquire })<_{P O}^{\alpha} e\right\}\end{array}$ \\
\hline Entry & $\begin{array}{l}D_{-}(\operatorname{sync}(i, s, \text { acquire }))=\left\{e: \exists r e l \mapsto s y n c(i, s, \text { acquire }) \text { and } e<_{P O}^{\alpha} \text { rel } \text { and }\right. \\
\text { e is tagged to } s\} \text {. } \\
D_{+}(\text {sync }(i, s, \text { acquire }))=\left\{e: \operatorname{sync}(i, s, \text { acquire })<{ }_{P O}^{\alpha} e \text { and } e \text { is tagged to }\right. \\
s\} .\end{array}$ \\
\hline Scope & $\begin{array}{l}D_{+}(\operatorname{sync}(i, s, \text { acquire }))=\left\{e: \text { sync }(i, s, \text { release })<_{P O}^{\alpha} e<_{P O}^{\alpha} \text { rel where rel }\right. \\
\text { corresponds with } \operatorname{sync}(i, s, \text { acquire })\} \\
D_{-}(\text {sync }(i, s, \text { release }))=\left\{\text { e }: \text { acq }<_{P O}^{\alpha} \text { e }<_{P O}^{\alpha} \text { sync }(i, s, \text { release }) \text { where acq }\right. \\
\text { corresponds with sync }(i, s, \text { release })\}\end{array}$ \\
\hline $\begin{array}{l}\text { All } \\
\text { Models }\end{array}$ & $\begin{array}{l}\text { must respect sync-well-formedness and } \forall i \in \mathcal{P} \forall x \in \mathcal{V}_{\mathcal{D}} \Rightarrow \\
\exists \beta \in \text { view }\left(\alpha,<_{\text {M sync }} \cup<_{P O}^{\alpha} \mid(i, \text { write }(, x,))\right): \beta \text { is serial. }\end{array}$ \\
\hline
\end{tabular}

Table 5. Characterization of synchronized memory models.

The order of successive stores by a processor to the same address, must however be respected".

Rule (2) forces ordinary accesses to be ordered before subsequent special accesses and rule (3) forces ordinary accesses to be ordered following previous special accesses. This implies that $D_{-}(s)$ must be defined as the ordinary accesses preceding $s$ in Process Order and $D_{+}(s)$ as the ordinary accesses following $s$ in PO.

(4) means that the basic coherence offered is Slow. Since the other synchronized are inspired in this one, we choose to maintain Slow as their basic coherence. In the case of the Release model, its original definition says that the basic coherence is Cache, but this detail is not emphasized by related works as [8]. Anyway, the relevant aspect of synchronized models in not the basic coher- 
ence, but the relations forced by synchronization operations and, in any case, our framework is capable to express that kind of requirements for the basic coherence. Then, the only thing that differentiates one model another is the definition of the sets $D_{-,+}(s)$. The orders that must be preserved for any view in any model are:

$-<_{P O}^{\alpha} \mid \mathcal{V}_{\mathcal{S}}$ : as stated by rule (1), accesses on synchronizing variables must be sequentially consistent: this ordering is not restricted to any particular process or variable (differing to Slow consistency requirement).

$-<_{D}^{\alpha}$ : ordering induced by synchronizations must be respected. It depends on the definition of $D_{-,+}(s)$ for each model.

$-<_{P O}^{\alpha} \mid(i$, write $(\cdot, x, \cdot))$ : this is the requirement for Slow consistency.

The first two orders establish the relations induced by the use of synchronizations. We group them in $<_{M E s y n c}^{\alpha}$ (see table 4).

Weak consistency was the first synchronized model proposed. It does not regard special accesses as primitives different of reads and writes. It only means that the shared memory system is able to distinguish between two types of variables and it can make variables of one type sequentially consistent, as induced by rule (1).

Release This model was introduced by Gharachorloo et al. in [12]. The definition is:

"A system is release consistent if (1) before an ordinary load (read) or store (write) is allowed to perform with respect to any other processor, all preceding acquire accesses must be performed, and (2) before a release access is allowed to perform to any other processor, all preceding ordinary load and store accesses must be performed, and (3) special accesses are processor consistent with respect to one another."

Sequential consistency is also accepted as the requirement stated by condition (3) for special accesses: [12]. This model derives from the Weak model. It regards special accesses as primitives. With relation to the Weak model, the ordinary accesses ordered by synchronizations varies allowing more concurrency because it does not force, for instance, that in the same process, an ordinary access be completed before a subsequent acquire. In this case, $D_{-}$(release) are the ordinary accesses PO preceding the release and $D_{+}$(acquire) are the ordinary accesses $\mathrm{PO}$ following the acquire. Figure 1 exemplify the differences between the Weak and Release models. Further variations to this model have been made. For example, Lazy Release model allows a write notification to be postponed with respect to a process until this one acquires the lock paired to the release PO following the write that generated the notification. In this case, $a$ is in $D_{-}(a c q)$ iff exists $r e l \mapsto a c q$ and $a<_{P O}^{\alpha} r e l$, for some rel. 
Entry This model was introduced in [7]:

"A critical section is bounded by a pair of synchronization accesses to a synchronization variable $s$. An acquire access occurs at the beginning of a critical section, and is used to gain access to a set of shared memory locations. A release access occurs at the end of the critical section, and is used to signal that access is available. [...] The synchronization variable $s$ controlling access to the critical section is said to guard the shared data $\left(D_{s}\right)$ which can be manipulated within the critical section. A memory system is entry consistent if, an acquire access of $s$ is not allowed to perform with respect to processor $p_{i}$ until all updates to $D_{s}$ have been performed with respect to $p_{i} . "$

This model is a generalization of Lazy Release consistency. Here, ordinary variables are tagged to synchronization variables. At an acquire, only ordinary variables tagged to the variable on which the acquire acts are made consistent. Here, $a$ is in $D_{-}(a c q)$ iff exists $r e l \mapsto a c q$ and $a<_{P O}^{\alpha}$ rel and $a$ is tagged to the variable on which both $a c q$ and rel acts.

Scope This model has been recently introduced [13]. It is similar to Entry consistency. However, the set of data actions controlled by a special synchronization is not associated with the synchronization variable, but with what they refer to as a session: the set of actions issued when holding a lock (between the acquire and the release). Each synchronization variable controls a consistency scope. The original definition is:

"Before a new session of a consistency scope is allowed to open at processor $\mathrm{P}$, any write previously performed with respect to that consistency scope must be performed with respect to P. A memory access issued by processor $\mathrm{P}$ is allowed to perform only after all consistency scope sessions previously opened by $\mathrm{P}$ have been successfully opened."

Here, $D_{-}$of an acquire are the ordinary accesses between it and the corresponding following release. Similarly, $D_{+}$of a release are the accesses between it and its corresponding acquire.

\section{Conclusions}

In this paper we have studied the formalization of synchronized memory coherency models, extending and modifying the formal framework introduced in [9]. The formalizations introduced permit us to compare different models among them, to rigorously prove properties about them and to prove the correctness of implementations of them $[10,4,5,3]$. 


\section{References}

1. M. Ahamad, R. Bazzi, R. John, P. Kohli, and G. Neiger. The power of processor consistency. Technical Report GIT-CC-92/34, College of Computing, Georgia Institute of Technology, December 1992.

2. M. Ahamad, J.E. Burns, G. Neiger, and P. Kohli. Causal memory: Definitions, implementation and programming. Technical Report GIT-CC-93/55, College of Computing, Georgia Institute of Technology, September 1993.

3. J. Bataller and J. Bernabeu. Building adaptable dsm systems. Technical Report II-DSIC-17/97, Dep. de Sistemas Informaticos y Computacion (DSIC), Polytechnic University of Valencia (Spain), 1997.

4. J. Bataller and J. Bernabeu. Characterizations of shared memory coherence. Technical Report II-DSIC-4/97, Dep. de Sistemas Informaticos y Computacion (DSIC), Polytechnic University of Valencia (Spain), January 1997.

5. J. Bataller and J. Bernabeu. Distributed causal memory algorithms. Technical Report II-DSIC-5/97, Dep. de Sistemas Informaticos y Computacion (DSIC), Polytechnic University of Valencia (Spain), 1997.

6. J.M. Bernabéu-Aubán and V. Cholvi-Juan. Formalizing memory coherency models. Journal of Computing and Information, 1(1):653-672, May 1994.

7. B.N. Bershad and M.J. Zekauskas. Midway: Shared memory parallel programming with entry consistency for distributed memory multiprocessors. Technical Report CMU-CS-91-170, Carnegie-Mellon University, September 1991.

8. J. B. Carter, J. K. Bennett, and W. Zwaenepoel. Techniques for reducing consistency-related communication in distributed shared memory systems. $A C M$ Transactions on Computer Systems, 13(3):205-243, August 1995.

9. V. Cholvi-Juan. Formalizing Memory Models. PhD thesis, Department of Computer Science, Polytechnic University of Valencia (Spain), December 1994. In Spanish.

10. V. Cholvi-Juan and J. Bernabeu Auban. Correctness proof for a distributed memory system. In Europar'96, pages 526-532, August 1996.

11. M. Dubois and c. Scheurich. Memory access dependencies in shared-memory multiprocessors. IEEE Transactions on Software Engineering, 16(6):660-674, June 1990.

12. K. Gharachorloo, D. Lenoski, J. Laudon, P. Gibbons, A. Gupta, and J. Hennessy. Memory consistency and event ordering in scalable shared-memory multiprocessors. In Proceedings of the 17th Annual International Symposium on Computer Architecture, pages 15-26. IEEE, April 1990.

13. L. Iftode, J.P. Singh, and K. Li. Scope consistency: A bridge between release consistency and entry consistency. Technical report, Dept. of Computer Science, Princeton Univ., 1996.

14. P. Kohli, G. Neiger, and M. Ahamad. A characterization of scalable shared memories. Technical Report CIT-CC-93/04, College of Computing, Georgia Institute of Technology, January 1993.

15. N. Lynch. I/O Automata: A model for discrete event system. Technical Report MIT/LCS/TM-351, Laboratory for Computer Science, Massachusetts Institute of Tecnology, March 1988. 